\title{
Proposal of forceps force limiter design using leaf spring buckling
}

\author{
Satsuya Noda ${ }^{1 *} \mathbb{D}$, Yasunori Tokuoka², Satoru Kuriu ${ }^{2}$ and Tadashi Ishida ${ }^{2}$
}

\begin{abstract}
To prevent accidents in minimally invasive surgeries, force limiters have been developed for forceps grippers. When a force limiter is in use, if the absolute value of its spring constant is reduced, the risk of damage to the organs decreases. This paper proposes the use of a leaf spring buckling mechanism as a force limiter for forceps. The results obtained indicate that the spring constant of a buckled leaf spring is lower than that of a normal coil spring. Furthermore, the use of a leaf spring allows the independent adjustment of its thickness and width, based on the stress and force values. This enables an easy calibration of the threshold value. In the experiments, the spring constant of the buckled leaf spring was $1.5 \times 10^{-1} \mathrm{~N} / \mathrm{mm}$, which is half of that of a normal coil spring. After calibrating the gripping force, it was confirmed that the force limiter reduced the extent of damage to the dummy organs in the ex vivo experiments.
\end{abstract}

Keywords: Leaf spring, Buckling, Forceps, Force limiter

\section{Introduction}

An advantage of minimally invasive surgery (MIS) is the reduction of damage to the patient after the surgery; however, this type of surgery requires difficult techniques by surgeons as compared to conventional surgery. The degree of freedom in general surgical instruments decreases from six to four because these instruments are inserted into trocars. Hence, surgical robots [1] or manipulators [2] have been developed.

One problem with these surgical instruments is the transmission of force information. Some surgical robots [1] lose the force information to the surgeons. Applying excess force in gripping or exclusion might cause serious damage to the organs. To prevent such accidents, it is generally effective to measure the force acting on the forceps; thus, many force sensors have been developed for forceps [3-5]. However, equipping forceps only with force sensors does not eliminate accidents owing to

\footnotetext{
*Correspondence: snoda@fukushima-nct.ac.jp

${ }^{1}$ Department of Mechanical System Engineering, National Institute of Technology, Fukushima College, 30 Tairakamiarakawa-aza-Nagao, Iwaki 970-8034, Fukushima, Japan

Full list of author information is available at the end of the article
}

the use of excess force. Furthermore, surgeons with little experience in MIS have difficulty controlling the force acting on the instruments, even if surgical manipulators with force feedback are used.

To prevent such accidents, force limiters for forceps [68] have been developed. If the gripping force exceeds the threshold value, the coil spring begins to deform, as shown in Fig. 1. Because a normal force limiter employs a linear coil spring, the gripping force increases proportionally to the handle displacement, which might cause damage to the gripped organ. On the other hand, if the spring force decreases with the displacement, the gripping force decreases, which might cause the organ to slip from the gripper. Therefore, to decrease the change in force with respect to the displacement, the absolute value of the spring constant of a force limiter should be low.

To reduce the spring constant without changing the threshold value of the force limiter, the number of windings of a coil spring should be increased. However, such springs will require more length and installing them for the gripper will become difficult. Although a constant load spring or a coil spring with a noncircular pulley [9] can be employed to reduce the spring constant, the size 


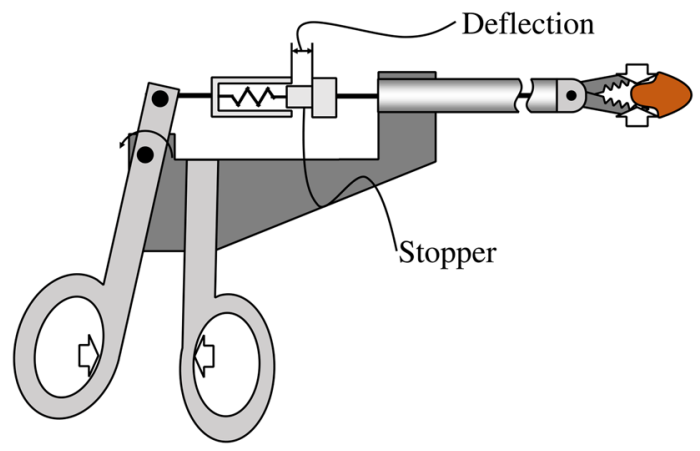

Fig. 1 General force limiter mechanism

of this spring will pose difficulties in attaching it to the gripper.

This paper proposes the use of a leaf spring buckling mechanism as a force limiter for forceps. The spring constant of the buckled leaf spring is lower than that of a normal coil spring. Previous studies have examined mechanisms that use buckling; for example, some personal computer keyboards employ buckled coil springs [10], leaf springs are used in constant load springs [11], and a force limiter for a toothbrush employs a leaf spring [12]. Compared to previous studies, the advantage of this study is the ease of adjusting the design parameters. The use of a leaf spring allows the independent adjustment of its thickness and width, based on the stress and force values. The aim of this research is to confirm the principle of the gripping force limitation using leaf spring buckling.

\section{Force limiter mechanism}

In the prototype model of the force limiter, the ease of a leaf spring attachment is significant. However, if the structure with a leaf spring as shown in Fig. 1 is adopted, it requires linear slider mechanisms, which results in larger size for the forceps.

Let us consider the mechanism of force limiter as shown in Fig. 2. The handle of the force limiter consists of base and movable handles. Generally, the movable handle is used for gripping, whereas the base handle does not move. If the gripper of the distal side grasps an organ and the movable handle rotates in the gripping direction, the gripping force increases. Hence, the gripping force will not increase if the gripper of the distal side does not rotate. This study proposes the base handle mechanism that moves only when the gripping force exceeds the threshold value. If the base handle can be driven by a constant force, the gripping force will not increase (see Fig. 2 (b)). The use of the leaf spring buckling enables to prevent the gripping force increase.

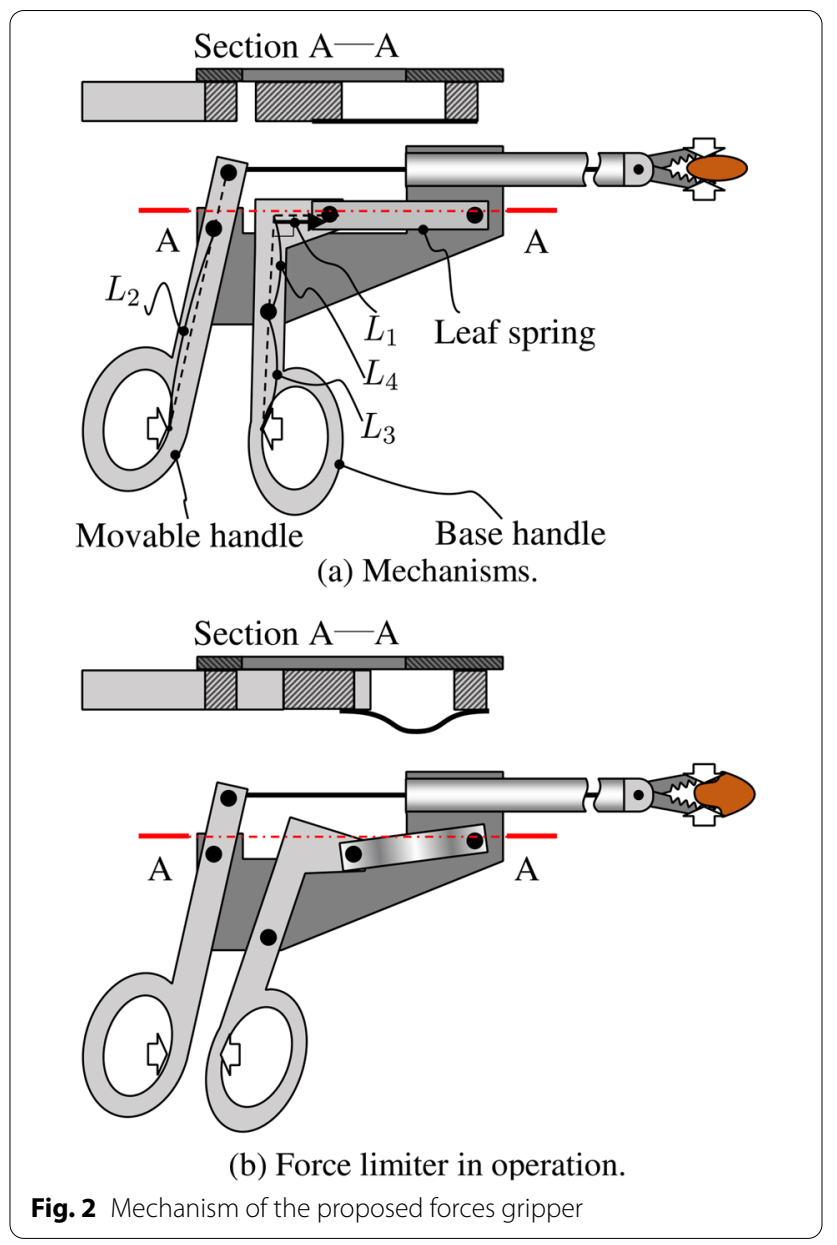

Furthermore, the linkage mechanism of the base handle also assists in preventing the gripping force increase.

\section{Leaf spring buckling}

Figure 3 shows the coordinate system of the leaf spring. The bending formula is given by the following expression:

$$
\kappa(s)=\theta^{\prime}(s)=-\frac{M(s)}{E I},
$$

where $s$ is the arc length from the edge, $x(s)$ and $y(s)$ indicate the position of the leaf spring at $s, \theta(s)$ is the angle of the tangential line from the $x$-axis at $s, '$ denotes the

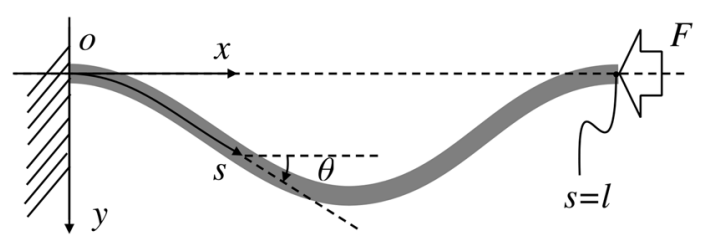

Fig. 3 Coordinate system of the buckled leaf spring 
derivative with respect to $s, \kappa(s)$ is the curvature at $s, M(s)$ is the bending moment at $s, E$ is the Young's modulus of the leaf spring, and $I$ is the moment of inertia of the cross-sectional area of the leaf spring.

Because the cross section of the leaf spring is a rectangle, $I$ is given by

$$
I=\frac{b t^{3}}{12},
$$

where $b$ and $t$ denote the width and thickness of the leaf spring, respectively.

$M^{\prime}(s)$ denotes the shearing force at $s$. By differentiating (1), the following equation is obtained:

$$
\begin{aligned}
& \frac{d^{2} \theta(s)}{d s^{2}}=-A \sin \theta \\
& A=\frac{F_{b}}{E I},
\end{aligned}
$$

where $F_{b}$ denotes the buckling load.

Equation (3) is analogous to the equation of motion of a nonlinear pendulum, whereby the buckling load corresponds to the period of the pendulum. Let $\theta_{\max }$ be $\operatorname{Max}|\theta(s)|$. It is known that the period of a nonlinear pendulum is given by the complete elliptic integral of the first kind and depends on $\theta_{\max }$ [13]. This study assumes $\theta_{\max } \leq 45^{\circ}$, as described in the Simulation section. In this range, the period error of a nonlinear pendulum is less than $5 \%$ of that of a linear pendulum. Similarly, the buckling load error of a nonlinear deformation is less than $10 \%$ of that of a linear deformation.

The buckling load of a linear deformation is given by the following equation:

$$
F_{b}=c \pi^{2} \frac{E I}{l^{2}},
$$

where $l$ denotes the length of the leaf spring and $c$ denotes the coefficient of fixity. From (2) and (5), it is obvious that $b$ can be used to adjust $F_{b}$ because $F_{b} \propto I \propto b$.

The coefficient $c$ is determined as follows:

(a) $c=1$, if both edges cannot support momentum loads (see Fig. 4 (A)).

(b) $c=4$, if both edges can support momentum loads (see Fig. 4 (B)). Because the proposed force limiter can support the moment of the buckling direction, as shown in Fig. 2, the procedure for this study sets $c=4$.

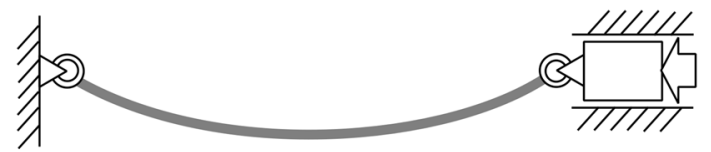

(a) Moment zero

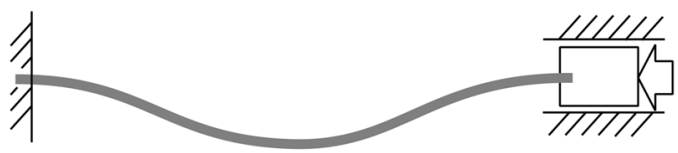

(b) Slope zero

Fig. 4 Fixation model of the leaf spring at the edge

\section{Design procedure for the force limiter mechanism}

Because the spring constant of the buckled leaf spring is lower than that of a normal coil spring, it is difficult to analyze the stress produced by the load. The analysis of this stress requires the calculation of the leaf spring deformation. Therefore, the parameters are determined in the order of $l, t$, and $b$, as shown in Fig. 5. Considering the handle size, the parameters were set so as to minimize $l$. A detailed description of each step in the design of the leaf spring is given below:

Step 1: Determine the range of $L_{3}$ and $L_{4}$.

Let $F_{\text {in }}$ be the input force to the linkage and $\Delta x_{\text {in }}$ be the base handle displacement in the $x$ direction. This step provides $F_{\text {in }}$ and $\Delta x_{\text {in }}$. The designed buckling load $F_{i}$ and displacement of the leaf spring $\Delta x_{i}$ are calculated using

$$
\begin{aligned}
& F_{i}=\frac{L_{3 i}}{L_{4 i}} F_{\mathrm{in}} \\
& \Delta x_{i}=\frac{L_{4 i}}{L_{3 i}} \Delta x_{\mathrm{in}} .
\end{aligned}
$$

$L_{3 i}$ and $L_{4 i}$ are determined using

$$
\begin{aligned}
& L_{3 i}=L_{3 \min }+\frac{L_{3 \max }-L_{3 \min }}{m_{1}} i \\
& L_{4 i}=L_{2}-L_{3 i},
\end{aligned}
$$

where $i=0,1 \ldots m_{1}$. In this range, the value of $L_{3}$ that minimized $l$ is determined.

Step 2: Determine the range of $l$.

Let $l_{i}$ be the value of $l$ that corresponds to $L_{3 i}$ and $L_{4 i}$. From the leaf spring length limitation, $l_{i}$ must satisfy $l_{i}>\Delta x_{i} . l_{\max }$ denotes the maximum length, which is limited by the handle size. Thus, $l_{i} \leq l_{\max }$. Hence, $l$ must be sampled in the range $\Delta x_{i}<l \leq l_{\max }$. Let $l_{i j}$ be 


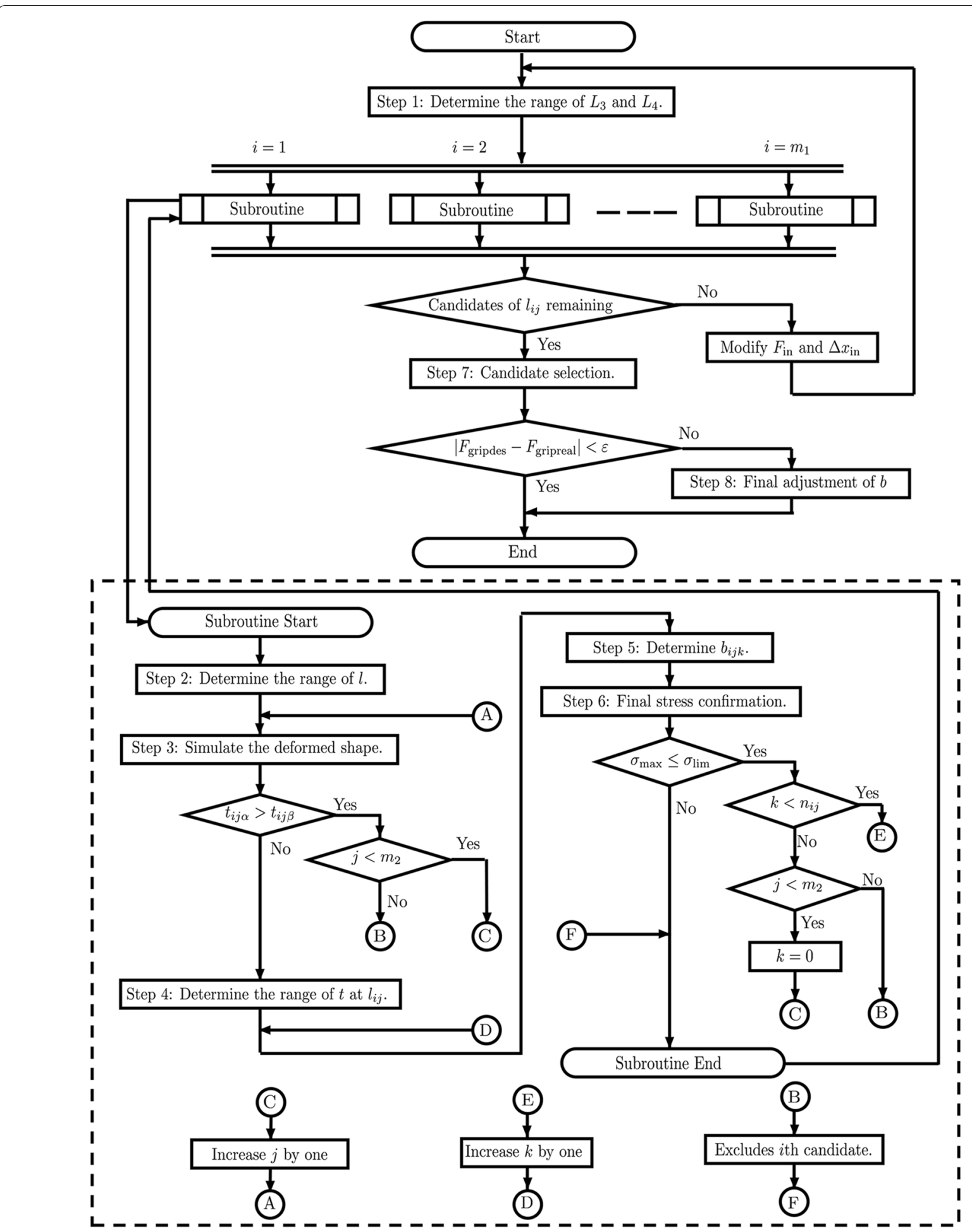

Fig. 5 Design procedure for each parameter 
the $j$ th sample of $l_{i}$. Then, $l_{i j}=\Delta x_{i}+\frac{l_{\max }-\Delta x_{i}}{m_{2}} j$, where $j=0,1 \ldots m_{2}$. In Step 2, $j=0$.

Step 3: Simulate the deformed shape.

The linear constitutive relationship is given by

$$
\left[\begin{array}{l}
x^{\prime}(s) \\
y^{\prime}(s)
\end{array}\right]=\left[\begin{array}{c}
\cos \theta(s) \\
\sin \theta(s)
\end{array}\right]
$$

where the initial conditions are as follows:

$$
\begin{aligned}
& (x(0), y(0))=(0,0) \\
& \theta(0)=\operatorname{atan} 2\left(y^{\prime}(0), x^{\prime}(0)\right) \\
& \left(x^{\prime}(0), y^{\prime}(0)\right)=(1,0) .
\end{aligned}
$$

The boundary conditions are as follows:

$$
\left(x\left(l_{i j}\right), y\left(l_{i j}\right)\right)=\left(l_{i j}-\Delta x_{i}, 0\right)
$$

Equations (3) and (10) are solved by a numerical method, such as the Runge-Kutta method. Let $A_{i j}$ be $A$ with respect to $l_{i j}$. To satisfy the boundary conditions, these calculations are nested within the adjustment procedure loop of $A_{i j}$ and $\kappa(0)$. To increase the convergence precision, the adjustment procedure is described as follows:

Loop 1: Let $\varepsilon$ be the allowable error of the edge position. The procedure adjusts $A_{i j}$, which satisfies $\left|y\left(l_{i j}\right)\right|<\varepsilon$. An optimization process is used to determine $A_{i j}$.

Loop 2: The procedure adjusts $\kappa(0)$, which satisfies $\left|x\left(l_{i j}\right)-\left(l_{i j}-\Delta x_{i}\right)\right|<\varepsilon$. If $\kappa(0)$ is updated, then Loop 1 is performed.

Step 4: Determine the range of $t$ at $l_{i j}$.

The constraints on $t$ are described as follows:

(a) The lower limit of $t$ Let $t_{\min }$ be the lower limit of $t$ and $t \geq t_{\mathrm{min}}$.

(b) The maximum stress

Let $\sigma(s)$ be the maximum stress at $s$, then $|\sigma(s)|$ is given by

$$
|\sigma(s)|=\frac{|M(s)|}{Z},
$$

where $Z$ denotes the modulus of the section, which is given by

$$
Z=\frac{b t^{2}}{6} \text {. }
$$

From (1), (2), (15), and (16), $\sigma(s)$ is calculated by the following equation that does not include $M(s)$ :

$$
|\sigma(s)|=\frac{|\kappa(s)| E t}{2} .
$$

According to (17), $\sigma(s)$ does not depend on $b$ but depends on $\kappa(s), E$, and $t$. Let $\sigma_{\text {lim }}$ be the proof stress. Because $|\sigma(s)| \leq \sigma_{\text {lim }}, t$ must satisfy

$$
t \leq \frac{2 \sigma_{\lim }}{E \kappa_{\max }},
$$

where

$$
\kappa_{\max }=\operatorname{Max}|\kappa(s)| .
$$

(c) The limitation of $b$

From (2) and (5), if $t$ is determined, then $b$ can be calculated by

$$
b=\frac{12 l_{i j}^{2} F_{i}}{c \pi^{2} E t^{3}} .
$$

Let $b_{\max }$ be the maximum $b$, which is determined by the gripper size. Then $b$ must satisfy $b \leq b_{\max }$, thus

$$
t \geq \sqrt[3]{\frac{12 l_{i j}^{2} F_{i}}{c \pi^{2} b_{\max } E}} .
$$

If $t>b$, then (20) cannot be used to determine $b$ because the buckling direction in this case is orthogonal to the direction shown in Fig. 2. Hence, to satisfy $t \leq b, t$ must satisfy

$$
t \leq \sqrt[4]{\frac{12 l_{i j}^{2} F_{i}}{c \pi^{2} E}} .
$$

Let $t_{i j k}$ be $t$ with respect to $l_{i j}$. Hence, $t_{i j k}$ must satisfy

$$
t_{i j \alpha} \leq t_{i j k} \leq t_{i j \beta},
$$

where $k=0,1 \ldots n_{i j}\left(n_{i j}<\left(t_{i j \beta}-t_{i j \alpha}\right) / \Delta t\right)$,

$$
t_{i j k}=t_{i j \beta}-k \Delta t
$$

$$
\begin{aligned}
t_{i j \alpha} & =\max \left(t_{\min }, \sqrt[3]{\frac{12 l_{i j}^{2} F_{i}}{c \pi^{2} b_{\max } E}}\right) \\
t_{i j \beta} & =\min \left(\frac{2 \sigma_{\lim }}{E \kappa_{\max }}, \sqrt[4]{\frac{12 l_{i j}^{2} F_{i}}{c \pi^{2} E}}\right) .
\end{aligned}
$$

The procedure sets $\Delta t=0.1 \mathrm{~mm}$, which is the thickness of a commonly available plate. In Step $3, k=0$. If $t_{i j \alpha}>t_{i j \beta}$, then the procedure is described as follows:

(a) If $j<m_{2}$, the procedure increases $j$ by one and returns to Step 3.

(b) If $j=m_{2}$, the procedure excludes $i$ th candidate. 
Step 5: Determine $b_{i j k}$.

Let $b_{i j k}$ be $b$ at $t_{i j k}$, then $b_{i j k}$ is calculated from (20).

Step 6: Final stress confirmation.

The maximum $|\sigma(s)|$ obtained by (17) is a little less than that obtained by the finite element method (FEM). Therefore, the final stress confirmation is conducted by the FEM. Let $\sigma_{\max }$ be the maximum stress obtained by the FEM. Because the leaf spring buckles symmetrically, the half-size analysis can decrease the calculation time. If $\sigma_{\max } \leq \sigma_{\text {lim }}$, then the leaf spring design is completed. If $\sigma_{\max }>\sigma_{\mathrm{lim}}$, then the procedure is described as follows:

(a) If $k<n_{i j}$, the procedure increases $k$ by one and returns to Step 5.

(b) If $k=n_{i j}$ and $j<m_{2}$, the procedure sets $k=0$, which increases $j$ by one and returns to Step 3 .

(c) If $k=n_{i j}$ and $j=m_{2}$, the procedure excludes $i$ th candidate.

Step 7: Candidate selection.

In this step, the minimum value of $l_{i j}$ is selected. If there are no candidates of $l_{i j}$ remaining, then the procedure returns to Step 1 and the values of $F_{\text {in }}$ and $\Delta x_{\text {in }}$ are modified.

Step 8: Final adjustment of $b$.

Let $F_{\text {gripreal }}$ and $F_{\text {gripreal }}$ be the designed and real gripping forces at the distal side, respectively. If $\left|F_{\text {gripdes }}-F_{\text {gripreal }}\right|>\varepsilon$, then the process must be repeated from Step 1 using modified $F_{\text {in }}$ and $\Delta x_{\text {in }}$ values. However, this would require remanufacturing the base handle, which is impractical. To avoid this, it is important to adjust the value of $b$. Let $\Delta b$ and $\Delta F_{\text {in }}$ be the differences between $b$ and $F_{\text {in }}$ values respectively. This study assumes $\left(F_{\text {gripdes }}-F_{\text {gripreal }}\right) \propto \Delta F_{\text {in }}$, and $\Delta b$ is given by

$$
\Delta b=\frac{12 l_{i j}^{2}}{c \pi^{2} E t^{3}}\left(F_{\text {gripdes }}-F_{\text {gripreal }}\right) \text {. }
$$

\section{Simulations and experiments Specification of the force limiter}

To verify the effectiveness of the proposed method, the forceps specifications were set as follows.

- Referring to the maximum range of the force sensor [14] for gripping the intestine, the gripping force of the force sensor was set to $F_{\text {in }}=6 \mathrm{~N}$.

- It was assumed that the gripping force of the distal side was equal to that of the proximal side due to the installation of a curved slider mechanism as will be described in the calibration section.
Based on the above specifications, the parameters were set and materials chosen as follows:

- The values of $l_{\max }$ and $b_{\max }$ were set to 180 and $30 \mathrm{~mm}$, respectively, as determined based on the length of the middle finger and half the length of the thumb, according to the database of Japanese hand sizes [15].

- The linkage parameters were set as $\left(L_{1}, L_{2}\right)=(2,60)$ mm.

- The range of $L_{3}$ was set as $\left(L_{3 \min }, L_{3 \max }\right)=(24,36)$ $\mathrm{mm}$. These values were determined based on the length of $L_{2} . \Delta x_{\text {in }}$ was set as $8.0 \mathrm{~mm}$.

- Strong surgical stainless steel (SUS631, with Young's modulus of $200 \mathrm{GPa}$, Poisson's ratio of 0.28 and $\sigma_{\lim }=1030 \mathrm{MPa}$ (proof stress)) was selected as the material for the leaf spring.

- The minimum thickness of the commonly available plate was set as $t_{\min }=0.1 \mathrm{~mm}$.

SolidWorks 2016 was used to conduct the FEM for the stress analysis.

\section{Spring constant of the buckled leaf spring.}

The spring constant of the buckled leaf spring was confirmed through simulations and experiments. Table 1 shows the candidates of $L_{3}$ and $L_{4}$. The value of $l$ was the smallest for the 4th candidate, i.e., $50 \mathrm{~mm}$. The linkage parameters were set as $\left(L_{3}, L_{4}\right)=(33,27) \mathrm{mm}$. Table 2 shows the specifications of the leaf spring. Figure 6 and Table 3 show the deformed shape of the leaf spring with $\Delta x_{i}=6.5 \mathrm{~mm}$; this simulation converged to $(x(l), y(l))=$ $(43.5,0) \mathrm{mm}$ and $\left(x^{\prime}(l), y^{\prime}(l)\right)=(1,0)$. Using the FEM, $\sigma_{\max }=997 \mathrm{MPa}$, which is lower than $\sigma_{\text {lim }}$ (see Fig. 7) . From Fig. 7, little difference of the stress was observed in the width direction.

Furthermore, the spring constant of the leaf spring was confirmed by experiments. Figure 8 shows the experimental setup for the force measurement. A force gauge (AIKOH model RZ-20) was attached on a linear stage,

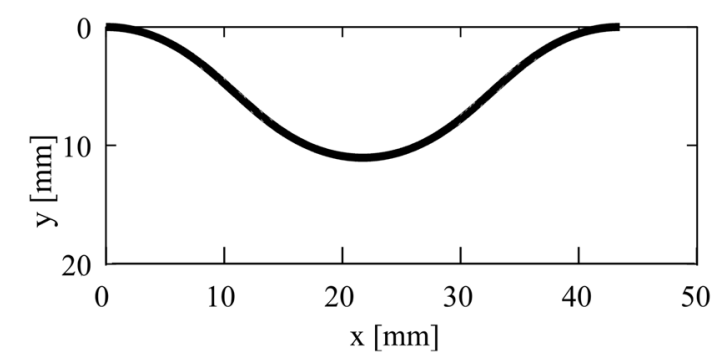

Fig. 6 Deformed shape of buckled leaf spring in simulation with $l_{i j}=50 \mathrm{~mm}$ and $\Delta x_{i}=6.5 \mathrm{~mm}$ 


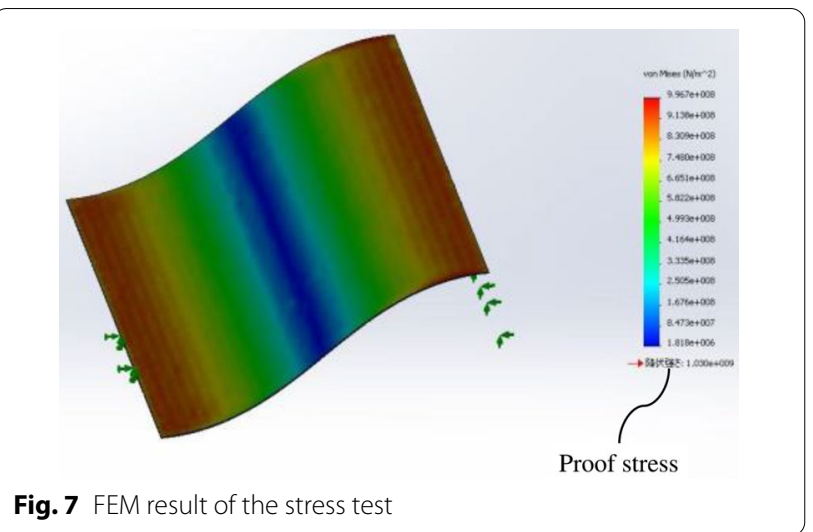

Fig. 7 FEM result of the stress test

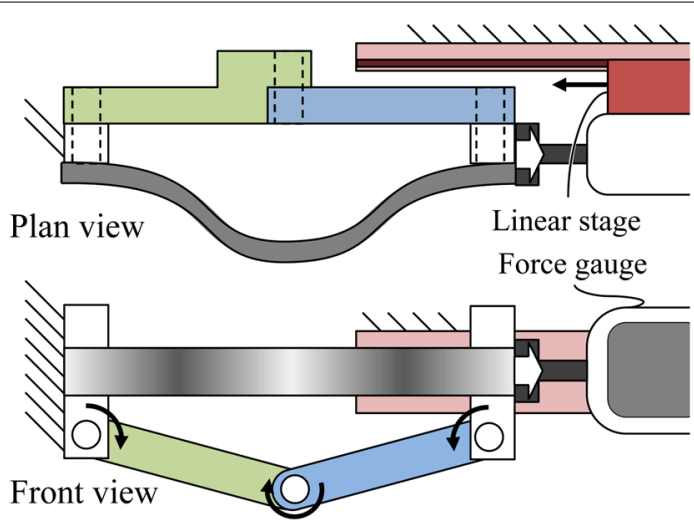

Fig. 8 Experimental setup for force measurement

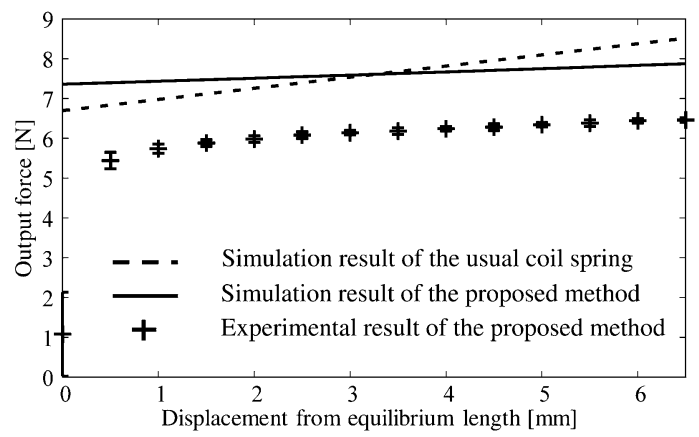

Fig. 9 Simulation and experimental result of buckling force with respect to displacement. Error bars show the standard deviation

which deformed the leaf spring. To separate the force gauge from the momentum load, the leaf spring was fixed to the arm device as shown in Fig. 8.

Figure 9 shows the result of the leaf spring buckling experiment. From the simulation results, the spring constant of the leaf spring was estimated to be $7.9 \times 10^{-2} \mathrm{~N} /$ $\mathrm{mm}$, which is about two-sevenths of that of a weak coil spring $\left(2.8 \times 10^{-1} \mathrm{~N} / \mathrm{mm}\right.$ for the MISUMI AWA 8-30

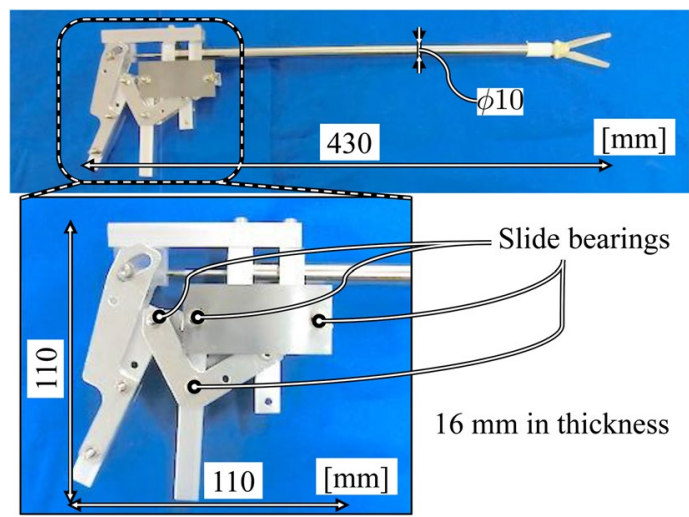

Fig. 10 Prototype force limiter

spring of outer diameter $8 \mathrm{~mm}$, length $50 \mathrm{~mm}$ at $6.7 \mathrm{~N}$, and maximum load of $8.53 \mathrm{~N}$ ). From the experimental results, the spring constant was estimated as $1.5 \times 10^{-1}$ $\mathrm{N} / \mathrm{mm}$ in the range $0.5 \leq \Delta x_{i} \leq 6.5 \mathrm{~mm}$ and $1.0 \times 10^{-1}$ $\mathrm{N} / \mathrm{mm}$ in the range $2.0 \leq \Delta x_{i} \leq 6.5 \mathrm{~mm}$. Although the experimental result was different from the simulation result, the spring constant of the buckled leaf spring was less than that of the weak coil spring.

Note 1: This procedure adjusted the value of $L_{1}$ to mini mize the absolute value of the spring constant of the leaf spring.

Note 2: In the experiments, a stage of a milling machine (TOYO ASSOCIATES: Little Milling 9) was used instead of a linear stage.

\section{Calibration of the gripping force limitation}

Figure 10 shows a prototype of the forceps with the force limiter. By using the curved slider mechanism of the proximal side, the displacement of the distal side gripper is equal to that of the proximal handle. To manufacture the prototype easily, the following material was selected:

- The proximal handle was built of aluminum alloy (A5052, A6063).

- The distal side gripper was built by 3D printing (Keyence: AGILISTA-3200, Material: AR-M2).

- Slide bearings (OILES \# 80 flange bush) were installed in the rotating parts.

Figure 11 shows the experimental setup for gripping force measurement. The handle of the proximal side is manually driven, and a force gauge measures the maximum force on the distal side of the gripper. This test was 

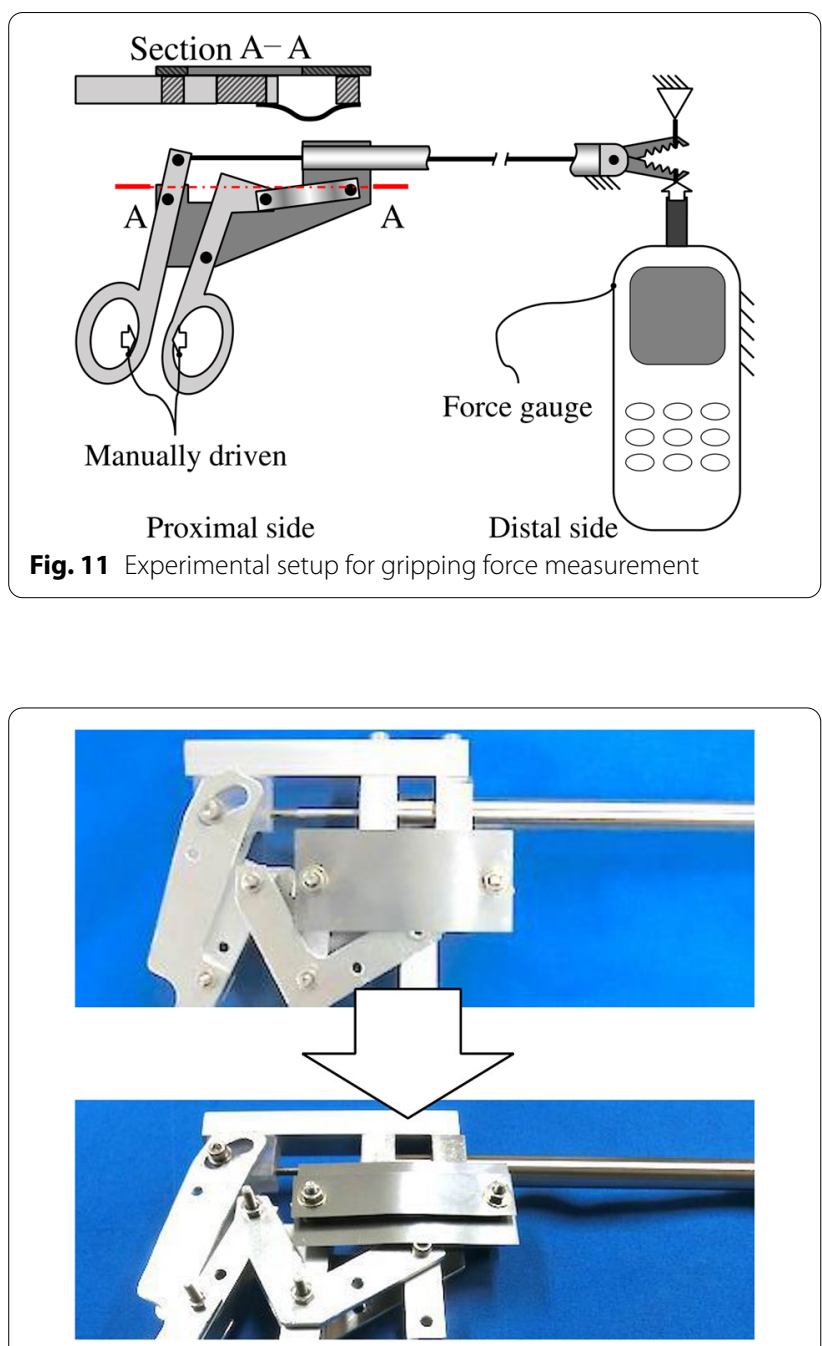

Fig. 12 Installation of additional leaf spring

performed 10 times, and the average gripping force was found to be $4.0 \mathrm{~N}$, which was about two-thirds of the theoretical value.

The variation can be attributed to friction and lack of rigidity in the transmitting mechanism. Friction at sliders and joints decreases the gripping force. Furthermore, because of the lack of rigidity, the phase of the slider is shifted, which also decreases the gripping force.

To increase the threshold value of the gripping force, let us consider adjustment of $b$, after assembling the mechanism. Referring to (20), $b$ should be set as $42 \mathrm{~mm}$, which is one and a half times larger than $28 \mathrm{~mm}$. Instead of adjusting $b$, the additional leaf spring $((l, b, t)=(50,14$, $0.1) \mathrm{mm}$ ) was installed in the gripper, as shown in Fig. 12. The gripping force increased to $6.1 \mathrm{~N}$, which was approximately the same as the theoretical value.

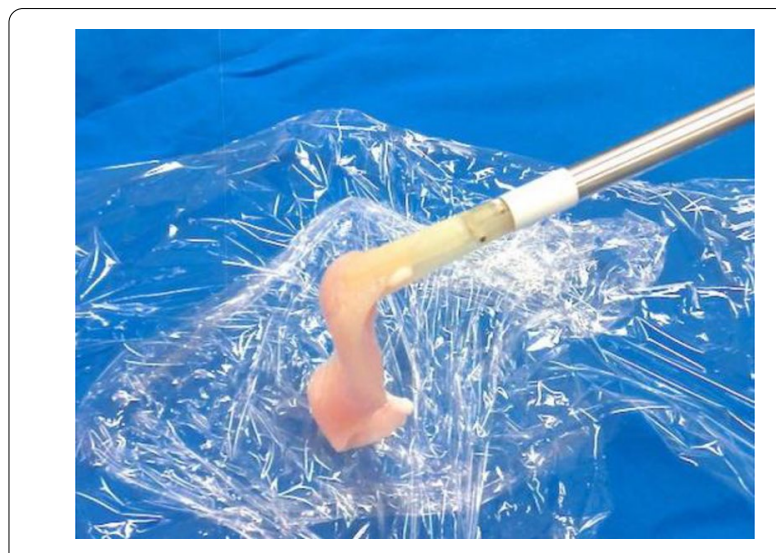

Fig. 13 Ex vivo experiments of the force limiter

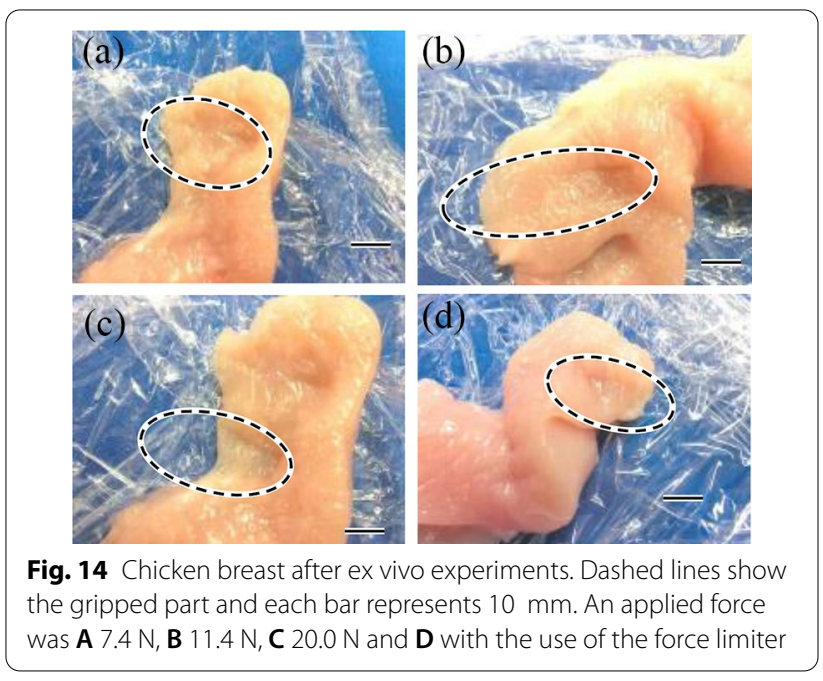

\section{Ex vivo experiments}

Ex vivo experiments were performed to confirm that the developed forceps could limit the gripping force. A piece of chicken breast (mass: $24 \mathrm{~g}$ ) was used as a dummy organ. In the preliminary experiments of chicken breast, the gripping scar was observed where the force was greater than $11.4 \mathrm{~N}$. This value was about twice as large as the maximum range of the force sensor [14]. The proximal side handle was manually driven until the force limiter began to function. For comparison, a force gauge (AIKOH model RZ-20) pushed the dummy organ by using the distal gripper part.

Figure 13 shows the results of the ex vivo experiments of gripping the chicken breast when the force limiter was functioning. No slips were observed during the experiments. Figure 14 shows the chicken breast after the gripping experiments. Figure 14 also shows the scars caused 

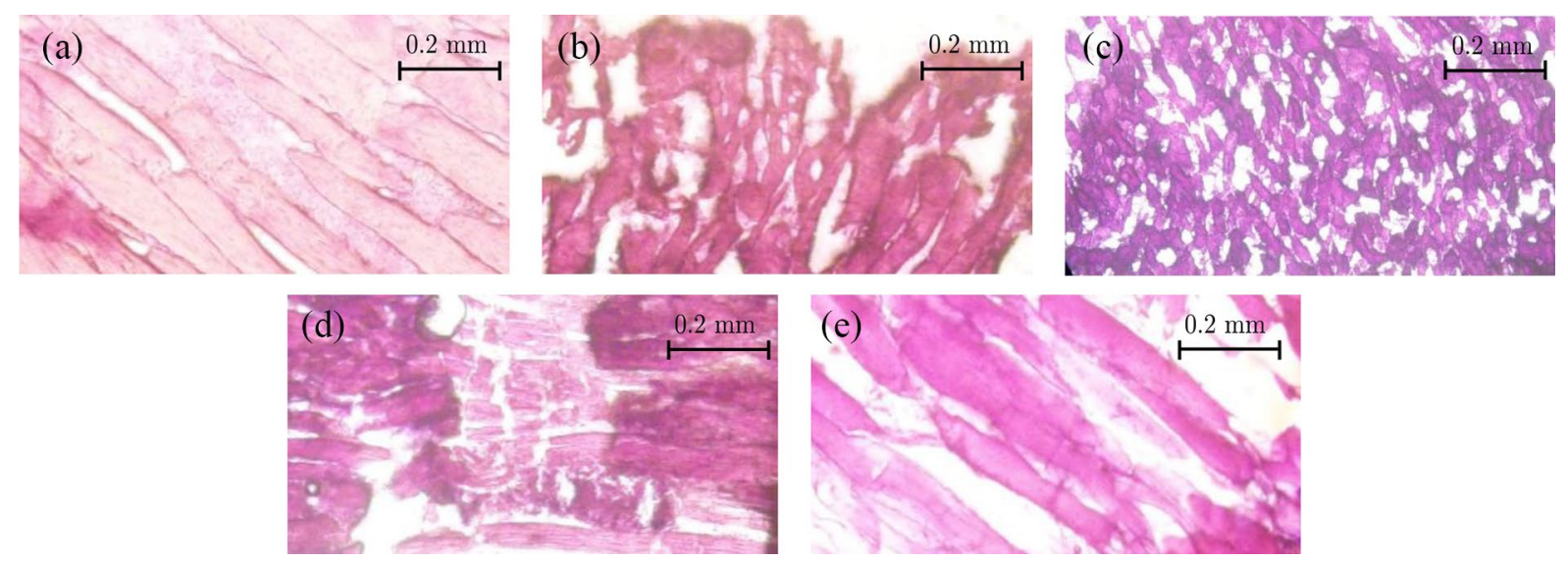

Fig. 15 Microscopic images of dummy organ tissue. An applied force was A 0 N, B 7.4 N, C 11.4 N, D 20.5 N and E with the use of the force limiter

by the force gauge, in the preliminary experiments. No scar to the dummy organ was observed in the gripping experiments, whereas a scar was observed where the force was greater than $11.4 \mathrm{~N}$.

Microscopic observations were performed to evaluate the damage to the dummy organs. The dummy organs were fixed in $4 \%$ paraformaldehyde for $10 \mathrm{~min}$, embedded in 3\% agar, and then frozen and sectioned in a cryostat $\left(18 \mu \mathrm{m}\right.$ thick, at $\left.-25^{\circ} \mathrm{C}\right)$. Prior to microscopic observations, the dummy organs were stained with hematoxylin-eosin.

Figure 15 shows microscopic images of the dummy organ. When no force was applied, the cells were $>0.3 \mathrm{~mm}$ in length (see Fig. 15A), whereas the gripper caused damage to the tissue when the applied force was $\geq 7.4 \mathrm{~N}$, which manifested as small voids $<100 \mu$ $\mathrm{m}$ in length (see Fig. 15B-D). The tissue was minimally damaged when the force limiter was implemented (see Fig. 15E). These results indicate that the proposed method can limit the gripping force.

\section{Discussion}

\section{Applied force at the handle}

In the ex vivo experiments, $F_{\text {in }}$ was measured during gripping. Figure 16 illustrates the experimental setup for measuring the $F_{\text {in }}$. The dummy organ was placed at the distal side of the gripper, and a force gauge was used to push the movable handle. For comparison, the applied force was measured when the gripper was locked. The $F_{\text {in }}$ on the dummy organ was $8.68 \mathrm{~N}$, whereas that of the locked gripper was $8.89 \mathrm{~N}$. The small difference in the values indicates that the gripping force applied to the dummy organ was $\sim 6.1 \mathrm{~N}$, as designed.

\section{Design of $F_{\text {in }}$}

In the ex vivo experiments, little damage to the dummy organs was observed when the force limiter was applied, while some damage was observed in the absence of the limiter when the force was $\geq 7.4 \mathrm{~N}$. These results indicate that when $F_{\text {in }}$ was set as $6.0 \mathrm{~N}$, the proposed method prevented damage to the dummy organs. Although these results are limited to the dummy organ, it is expected that the proposed method would prevent damage to other organs if $F_{\text {in }}$ is properly adjusted.

\section{Spring constant of the proposed method}

The spring constant of the buckled leaf spring was estimated as $1.5 \times 10^{-1} \mathrm{~N} / \mathrm{mm}$ in the range $0.5 \leq \Delta x \leq 6.5 \mathrm{~mm}$. These results indicate that the spring constant of the proposed force limiter is less than that of a normal coil spring. Although this study examined a

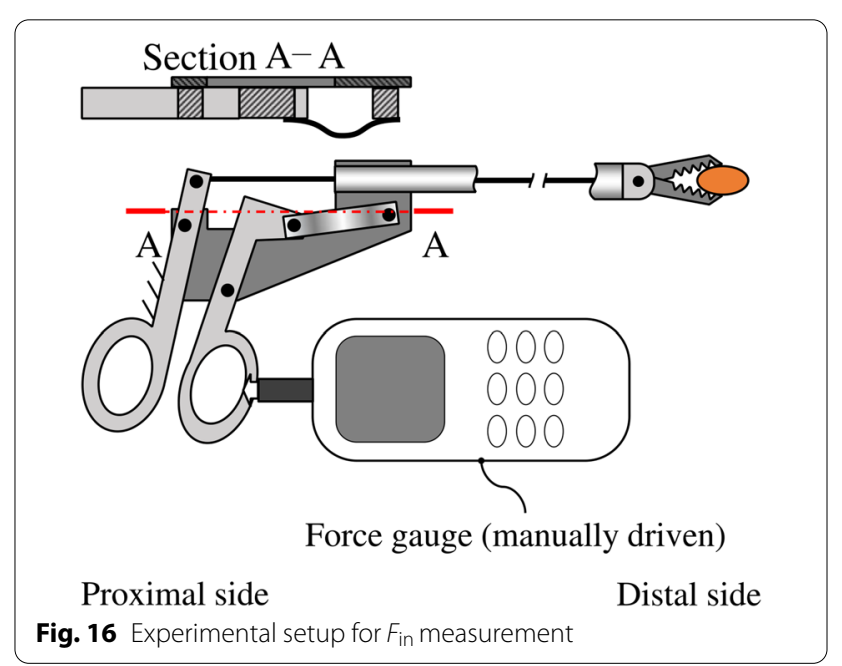




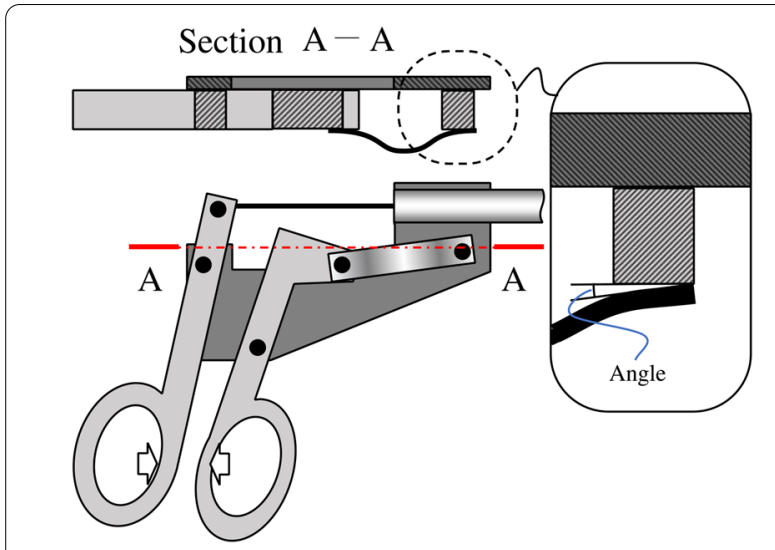

Fig. 17 Edge of the leaf spring

manual manipulator's grip, the proposed method can be applied to the joints of a surgical manipulator.

Discrepancy of the buckling force and the spring constant In the $0.5 \leq \Delta x \leq 6.5 \mathrm{~mm}$ range, the experimentally obtained spring constant was larger than that obtained via simulation. On the other hand, the buckling load was lower in the experiment than in the simulation, as shown in Fig. 9. Although these discrepancies occurred, the spring constant of the proposed method is lower than that of a weak coil spring, and the disparity in the buckling load is adjustable in Step 8.

These discrepancies can be attributed to the fixation method applied at the edges of the leaf spring. Owing to the lack of rigidity and backlash of the bearings, the angle at the edge is not always zero, as shown in Fig. 17. Both edges cannot support momentum loads when $\Delta x$ is lower than a specific value, whereas they can support momentum loads when $\Delta x$ is larger than a specific value, resulting in a discrepancy between the simulated and experimentally obtained spring constants. The angle at the edge increases the imaginary length of the leaf springs, decreasing the buckling load and the spring constant.

\section{Gripping position at the handle}

In the proposed mechanism, the threshold value of the force limiter depends on the gripping position of the base and the movable handle. Thus, the threshold value error arises from the gripping position error of the handles. To reduce the threshold value error, it is expected that the handle shape reduces the gripping position error. Owing to the ease of attaching the experimental tools, the developed handle shape is straight, as shown in Fig. 10. Therefore, to reduce the gripping position error, the handle shape should be improved, as shown in Fig. 2.

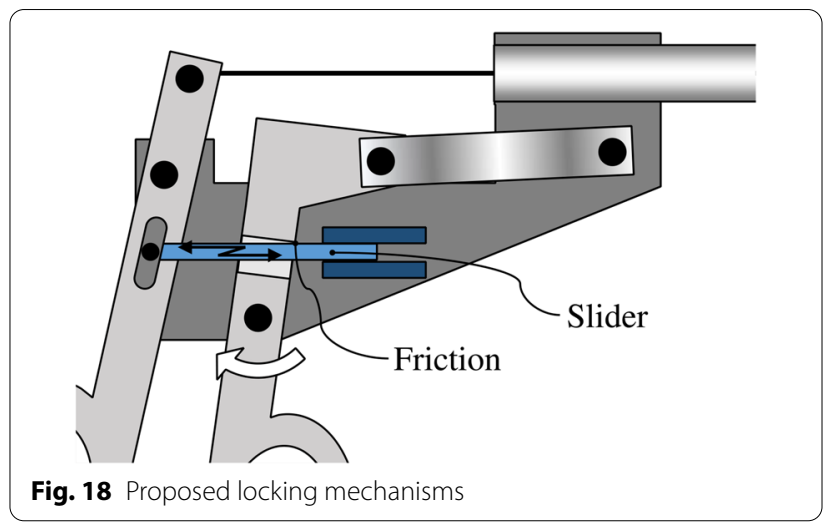

Table 1 Candidates of $L_{3}$ and $L_{4}$

\begin{tabular}{llll}
\hline Candidate No. & $\boldsymbol{L}_{\mathbf{3}}[\mathbf{m m}]$ & $\boldsymbol{L}_{\mathbf{4}}[\mathrm{mm}]$ & $\boldsymbol{I}[\mathbf{m m}]$ \\
\hline 1st candidate & 24 & 36 & 70 \\
2nd candidate & 27 & 33 & 60 \\
3rd candidate & 30 & 30 & 90 \\
4th candidate & 33 & 27 & 50 \\
5th candidate & 36 & 24 & 90 \\
\hline
\end{tabular}

Table 2 Design specification of leaf spring for the 4th candidate

\begin{tabular}{lll}
\hline Item & & Value \\
\hline$\Delta x_{i}$ & {$[\mathrm{~mm}]$} & 6.5 \\
$F_{i}$ & {$[\mathrm{~N}]$} & 7.37 \\
$\left(t_{i j \alpha}, t_{i j \beta}\right)$ & {$[\mathrm{mm}]$} & $(0.10,0.11)$ \\
$\left(l_{i j}, b, t\right)$ & {$[\mathrm{mm}]$} & $(50,28,0.1)$ \\
\hline
\end{tabular}

Table 3 Simulation result of buckled leaf spring

\begin{tabular}{lll}
\hline Item & & Value \\
\hline$\left(x\left(I_{i j} / 2\right), y\left(I_{i j} / 2\right)\right)$ & {$[\mathrm{mm}]$} & $(21.7,11.0)$ \\
$\left(x\left(I_{i j}\right), y\left(I_{i j}\right)\right)$ & {$[\mathrm{mm}]$} & $(43.5,0)$ \\
$\theta_{\max }$ & $\left.{ }^{\circ}\right]$ & 42.2 \\
$\left(x^{\prime}\left(I_{i j}\right), y^{\prime}\left(I_{i j}\right)\right)$ & & $(1,0)$ \\
\hline
\end{tabular}

\section{Limitation of $\sigma_{\max }$}

To satisfy $\sigma_{\max }<\sigma_{\text {lim }}$, the base handle should employ a displacement limiter. Therefore, a locking mechanism can be used as the displacement limiter. Figure 18 shows the proposed locking mechanism, which is based on a traditional Japanese pothook, Jizaikagi [16]. If the displacement of the base handle exceeds the threshold value, the rod comes into contact with the base handle. The friction caused by this contact 
provides the locking mechanism, and the locking force increases with the gripping force at the handles. The authors developed and confirmed its mechanisms in experiments [16].

\section{Conclusions}

To decrease the change in the force with respect to the displacement, the absolute value of the spring constant of a force limiter should be reduced. This paper proposed the use of a leaf spring buckling mechanism as a force limiter for forceps. The advantage of this study is the ease of adjusting the designed parameters. Specifically, the use of a leaf spring allows the thickness and width to be adjusted to the stress and force independently. Based on the simulations and experiments performed, the following findings were confirmed:

- From the simulation, the spring constant of the leaf spring was estimated as $7.9 \times 10^{-2} \mathrm{~N} / \mathrm{mm}$, which was two-sevenths that of the weak coil spring (MISUMI AWA 8-30).

- From the experiments, the spring constant of the buckled leaf spring was estimated as $1.5 \times 10^{-1} \mathrm{~N} /$ $\mathrm{mm}$.

- In the calibration stage, an additional leaf spring was installed in the force limiter. This caused the threshold value of the force limiter to increase from 4.0 to $6.1 \mathrm{~N}$.

- In the ex vivo experiments, minor damage to the dummy organ was observed with a microscope.

These results indicate that the spring constant of the proposed force limiter is lower than that of a normal coil spring and the use of leaf spring can reduce the damage caused to the dummy organ. Additionally, locking mechanisms that can be used to limit the stress in the leaf spring, were considered. Future studies will focus on improving the force limiter mechanism and performing in vivo experiments.

\section{Acknowledgements}

We would like to thank Editage (www.editage.com) for English language editing.

\section{Authors' contributions}

All work except ex vivo experiments was conducted by SN. In the ex vivo experiments, YT performed HE staining and microscopic observation. Analysis of HE staining and microscopic observation was conducted SK and TI. The other work about the ex vivo experiments was performed by SN. All authors read and approved the final manuscript.

\section{Funding}

Not applicable.

\section{Availability of data and materials}

The datasets supporting the conclusions of this article are included within the article.

\section{Declarations}

Competing interests

The authors declare that he has no competing interests.

\section{Author details}

${ }^{1}$ Department of Mechanical System Engineering, National Institute of Technology, Fukushima College, 30 Tairakamiarakawa-aza-Nagao, Iwaki 970-8034, Fukushima, Japan. ${ }^{2}$ Department of Mechanical Engineering, School of Engineering, Tokyo Institute of Technology, 4259, Nagatsuta-cho, Midori-ku, Yokohama-shi 226-8502, Kanagawa, Japan.

Received: 19 March 2020 Accepted: 22 January 2022

Published online: 14 February 2022

References

1. Bodner J, Wykypiel H, Wetscher G, Schmid T (2004) First experiences with the da vinci operating robot in thoracic surgery. Eur J Cardio-Thorac Surg 25(5):844-851

2. Frede T, Hammady A, Klein J, Teber D, Inaki N, Waseda M, Buess G, Rassweiler J (2007) The radius surgical system-a new device for complex minimally invasive procedures in urology? Eur Urol 51(4):1015-1022

3. Peirs J, Clijnen J, Reynaerts D, Van Brussel H, Herijgers P, Corteville B, Boone $S$ (2004) A micro optical force sensor for force feedback during minimally invasive robotic surgery. Sens Actuators A Phys 115(2):447-455

4. Puangmali P, Liu H, Seneviratne LD, Dasgupta P, Althoefer K (2012) Miniature 3-axis distal force sensor for minimally invasive surgical palpation. IEEE/ASME Trans Mech 17(4):646-656

5. Wagner CR, Stylopoulos N, Howe RD (2002) The role of force feedback in surgery: analysis of blunt dissection. In: Haptic Interfaces for Virtual Environment and Teleoperator Systems, 2002. In: 2002 proceedings 10th symposium on HAPTICS, pp 68-74. https://doi.org/10.1109/HAPTIC.2002.998943

6. Heimberger R, Schaumann U (1996) Forceps. Google Patents. US Patent 5,562,699. https://www.google.com/patents/US5562699

7. Sakaguchi Y, Sato T, Yutaka Y, Muranishi Y, Komatsu T, Yoshizawa A, Nakajima N, Nakamura T, Date H (2018) Development of novel force-limiting grasping forceps with a simple mechanism. Eur J Cardio-Thorac Surg 54(6):1004-1012

8. Michelini J, Uspenski A, Streiff A, John KR (2017) Grasper. Google Patents. US Patent App. 29/521,712

9. Endo G, Yamada H, Yajima A, Ogata M, Hirose S (2010) A weight compensation mechanism with a non-circular pulley and a spring: application to a parallel four-bar linkage arm(in japanese). J Robot Soc Jpn 28(1):77-84

10. Harris RH (1978) Buckling spring torsional snap actuator. Google Patents. US Patent 4,118,611 . https://www.google.com/patents/US4118611

11. Lan C-C, Wang J-H, Chen Y-H (2010) A compliant constant-force mechanism for adaptive robot end-effector operations. In: 2010 IEEE international conference on robotics and automation (ICRA), pp. 2131-2136. IEEE

12. Slocum J, Kamrin K, Slocum A (2019) A buckling flexure-based forcelimiting mechanism. J Mech Robot 11(4):041004

13. Beléndez A, Pascual C, Méndez D, Beléndez T, Neipp C (2007) Exact solution for the nonlinear pendulum. Revista brasileira de ensino de física 29(4):645-648

14. Takaki T, Omasa Y, Ishii I, Kawahara T, Okajima M (2010) Force visualization mechanism using a moiré fringe applied to endoscopic surgical instruments. In: 2010 IEEE international conference on robotics and automation, pp. 3648-3653. https://doi.org/10.1109/ROBOT.2010.5509708

15. Kawauchi M (2012) AIST database of Japanese hand size (in Japanese). https://unit.aist.go.jp/hiri/dhrg/ja/dhdb/hand/index.html

16. Noda S (2019) Development of lock mechanisms using jizaikagi mechanisms for forceps force limiter. In: Proceedings of the 2019 JSME conference on robotics and mechatronics, pp. 1-107. JSME

\section{Publisher's Note}

Springer Nature remains neutral with regard to jurisdictional claims in published maps and institutional affiliations. 